\title{
TRANSFORMATIONS OF A SYSTEM STATE UNDER THE SEQUENCE OF CONDITIONS
}

\author{
Yuri Andrianov ${ }^{1 *}$, Alexandr Kapustin ${ }^{1}$, Igor Kudryavtsev ${ }^{1}$, Petr Fishchenko ${ }^{1}$ \\ ${ }^{1}$ Volga State University of Technology, Russia
}

The algorithm of transformation of a system states is established on the basis of applying the methods of mathematical modelling and theoretical mechanics, providing preservation of a stationary property of the system. The form and the sizes of dangerous zones for a moving exosystem (a person in a medical exoskeleton) are determined as well. This will allow to producethe maximum allowable velocity, avoiding both lateral overturning of the exosystem, and collision with obstaclesduring its movement.

Key words: Stationary property of a system, Systemstates, Maximum allowable velocity, A person in a medical exoskeleton, Lateral stability condition, Collision with an obstacle

\section{INTRODUCTION}

In March 2017 the Volga State University of Technology (VSUT)won the competition within the framework of the program of support of leading Russian highereducational establishments, decision No. 218 Open competition on selection of the organizations to have the right of receiving grants for implementation of complex projects on creation of hi-tech manufacture with the project Creation of hi-tech manufacture of multipurpose robotized medical exoskeleton (REM) [1].

Exoskeleton (translated from the Greek as 'external skeleton') is a mechanism with mobile junction of the parts, similar to a human being joints, moving together with a human body it is attached to [5]. Exoskeletons provide people with superhuman force, and they give the opportunity to walk for people with health limitations [6-14]. A large number of works are devoted to solving the problem of stability of a person in an exoskeleton, some recent ones, havingRSCl, are [15-18].

We shall call the person-exoskeleton system as an exosystem. It should possess a 'stationary property of a system', i.e. it should not fall sideways neither when moving, nor in the rest position, and it should not collide with an obstacle during movement. Displacement of the center of gravity can be considered as one of the ways of maintaining stability of a person-exoskeleton system. The basic attributes of action of the given technical decision are in the fact that the system of stabilizing represents arotary-support device with an opportunity of displacement of the center of gravity of useful loading against the direction of movement by means of interlocked sloped drive rings with an opportunity of their relative turn. The person-exoskeleton systemcan be modelled by a simplified material point located in the center of gravity of this system which in turn is located at some $\mathrm{H}$ heightabove a horizontal base platform. Then it is possible to show, that there are such two dangerous zones in uniform move- ment (walking or running) entering which can lead to serious undesirable consequences. The size of the first and the second dangerous zones are determined in this work. Avoiding entering such dangerous zones (dangerous situations) it is possible to achieve a 'stationary property of a system'.

One of the advantages of the exosystemis its lateral stability, i.e. ability of the person in the exoskeleton not to fall down or sideways when getting a small cargo, moving and unloading. However it is necessary to provide this stability, meeting the condition of lateral stability [4]. Besides, when movingthe exosystem can come across an obstacle, which needs stopping without colliding or arrangingthe bypassing, not touching the obstacle.

Work objective is to define the form and size of dangerous zones to beexcluded from the area of exosystemmovement. It will allow to reach maximum allowable velocity, avoiding lateral overturning of the person in an exoskeleton when moving, taking into account a lateral stability condition [4]. In case of an obstacle in a straightline trajectory of movement, the estimation of an opportunity either to stop, not colliding with an obstacle, or 'to evade', turning aside and not falling sideways, is made. The paper offers

1) an algorithm (method) for consecutive changeover (transition) of a system from the current condition into next state, constant 'stationary property of the system' being unchanged;

2) implementation of the method given in the task of safe exploitation of a medical exoskeleton,'stationary property of the system' implying excludingthe object entering two dangerous zones, i.e.avoidance of lateral overturning and collision with an obstacle;

3) dimensioning the first dangerous zone and working out practical recommendations on lateral stability support; 
4) dimensioning the second dangerous zone and working outpractical recommendations on avoiding collision with an obstacle.

It is required to provide such a change of conditions and

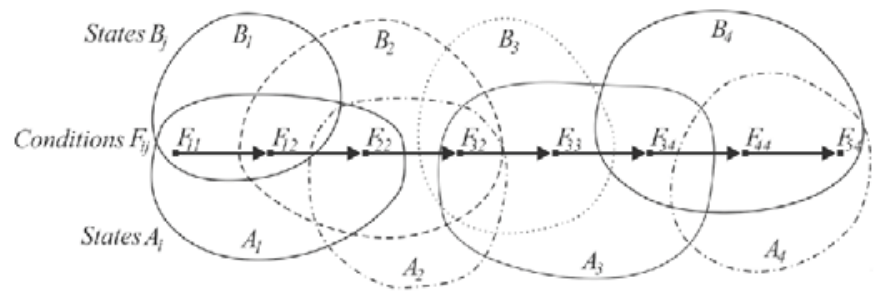

Figure 1: The algorithm of theexosystem change-over into next state

such change-overs of the system from its current state into next one that a system preset propertyCwas carried out continuously, being a 'stationary property of the system'.

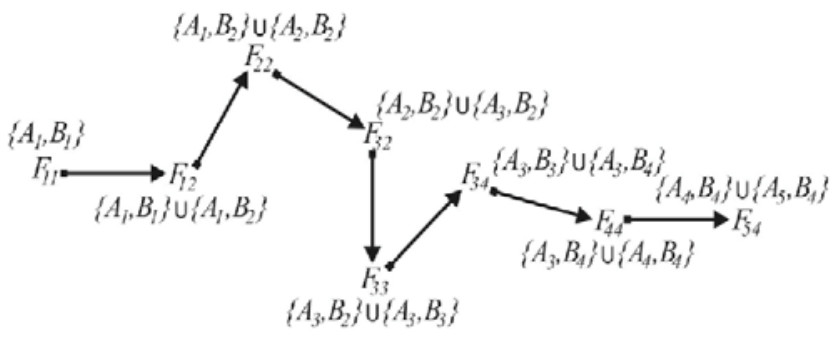

Figure 2: The order of occurrence and removal of 'pairs of conditions' and system states corresponding to them

In order for the system to possess property $C$, the following algorithm (method) of transition into next state is offered. First, conditions $\{A 1, B 1\}$ are imposed on the exosystem and the system, getting into condition F11, obtains property $\mathrm{C}$ which should not be lost further (Fig. 1 and Fig. 2.). As the exosystemcan successively be in two states at every 'pair of conditions', it should be transferred intothe 'second' conditionF12, not removing conditions $\{A 1, B 1\}$. Conditions $\{A 1, B 2\}$ are added to currentones. As the 'second'state of the system takes place under the same conditions $\{A 1, B 2\}$ as next state, conditions $\{A 2, B 2\}$ are added first and then the system should be transferredinto the 'third' state F22. After that, conditions $\{A 1, B 1\}$ are removed. The process proceeds similarly. Under conditions $\{A 2, B 2\}$ the system should be transferred from the 'third' state F22 into next, the 'fourth' state F32. As the 'fourth' state of the system takes place under conditions $\{$ A3, B2 $\}$ and under the same conditions as next state, conditions $\{A 3, B 3\}$ are added firstand then the system should be changed over into the 'fifth' state F33. Adding conditions $\{A 3, B 4\}$, we change the system over into state $F 34$ (conditions $\{A 2, B 2\}$ are removed). Adding $\{A 4, B 4\}$, we change the system over into state F44 (conditions $\{A 3, B 3\}$ are removed). Adding $\{A 5, B 4\}$, we change the system over into state F54 (conditions $\{A 3, B 4\}$ are removed). Andsoon. As any of the 'next pair conditions' are imposed on the system each time, the objectis provided with property Cpossession. Implementation of this algorithm is of interest for solving the following specific task. To solve the problem under study the methods of mathematical modelling and theoretical mechanics were applied.

\section{EXPERIMENTAL SECTION.}

As a result of preliminary design and engineering work an exoskeleton prototype was developed (Fig. 3). The design includes: 1 - lumbar module for breadthwise adjustment; 2 - control panel; 3 - system of power supplies; 4 -support handle; 5 - system of lumbar spine fixing; 6 phastexof waist beltsadjustment; 7 - stop; 8 -top flexible cable; 9 - hip joint electric drive; 10 - protective cover; 11 -hip holder; 12 -hip joint module adjustment in length unit; 13 - bottom flexible cable; 14 -knee hingeelectric drive; 15 and 17 -cnemisholder; 16 -cnemismodule adjustment in lengthunit; 18 -calxfixer; 19 - foot fastening collar; 20 -flexure hinge; 21 - sole with gauges
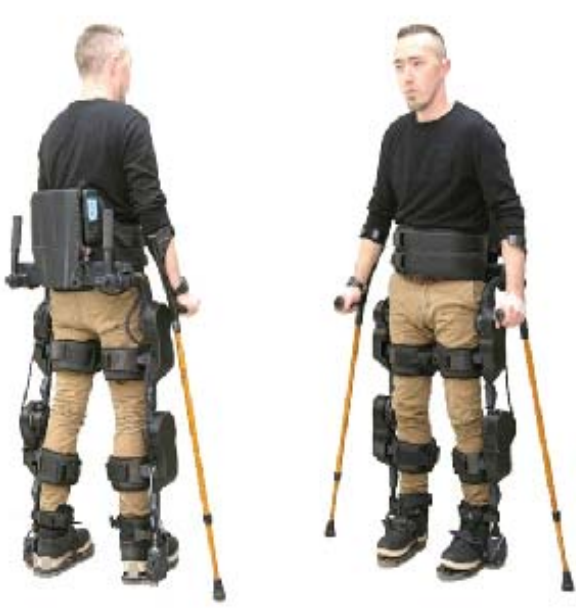

2

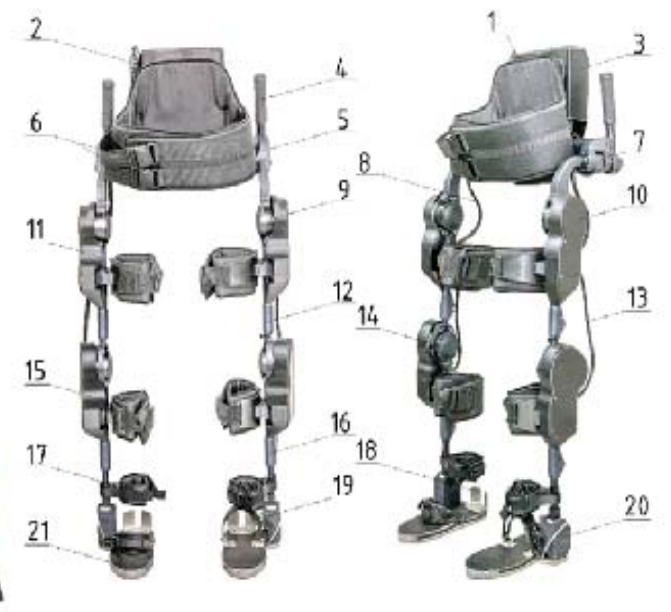

Figure 3: An exoskeleton general view 
Given $\mathrm{m}$, the weight of the whole system (the weight of the person together with the weight of the exoskeleton and the weight of a cargo); L, the width of a baseplatform; $\mathrm{H}$,the height of arrangement of the center of gravity of the whole exosystemabove thebase platform;f, the factor of friction between the soles and the surface in case of stopping, it was targeted to receive a condition for definingmaximum allowable velocityand to find the form of two dangerous zones where overturning of the exosystemsideways or collision with an obstacle is possible, as well as to find the dimensions of those dangerous zones.

\section{RESULTS AND DISCUSS}

Let us assume that a trajectory of movement of the exosystemis some flat curve in a horizontal plane. To simplify problem solving we consider uniform movement, i.e. movement atvelocitywhich is constant in absolute value. Velocity is a vector variable characterized by both number, and direction. As the velocity modulus remains constant and the direction of velocityvector remains random, the trajectory remains curvilinear. Any curve can be divided into parts. The more it is divided, the more the parts are similar to arcs of circles of different diameters. It is possible to consider circumferential movement along one of such circles. In this work we consider the case of uniform circumferential movement when a condition $|v|=$ const and $\mathrm{R}=$ const is met. The person with an exoskeletonand a cargo is modelled as a material point having weightmand body force $P=m g$, where $g$ is gravity factor. Under uniform circumferential movement tangential acceleration atis defined as a derivative of velocity against time. As the velocity of movement is constant in absolute value, both tangential and angular acceleration equal zero. The value of normal accelerationanis defined as the relation of squaredvelocity to the radius of curvature of a trajectory in the point of contact. Under circumferentialmovement the radius of curvature of a trajectory equalsthe radius of the circle. Full acceleration is the sum of vectors of rotary and centripetal acceleration. As rotary acceleration equals zero, full acceleration coincides with centripetal one (Fig. 4).

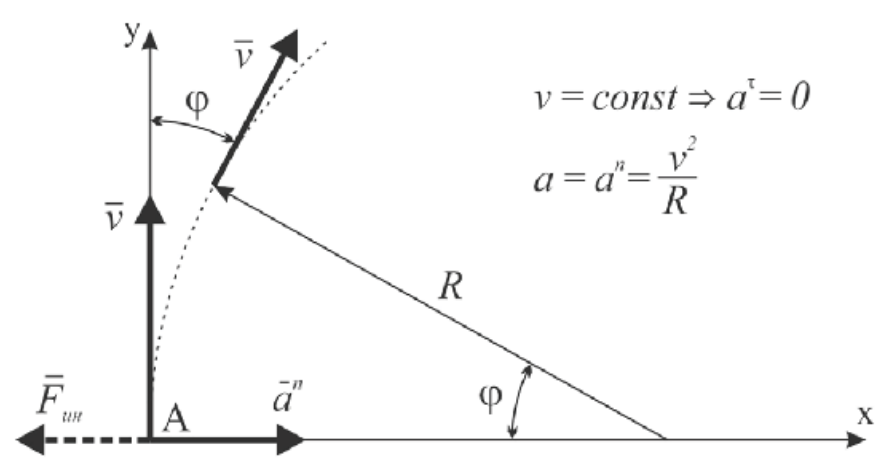

Figure 4: Acceleration directions and g-force
The force of inertia applied in the center of weights of the whole system (a person and an exoskeleton, probably together with a small cargo) equals in value to the product of the system weight multiplied by full acceleration and is directed opposite this acceleration.

As this force of inertia is directed horizontally at some heightHabove the horizontal base platform, this force creates 'overturning moment'. The 'overturning moment' tends to incline and overturn the exosystemsideways round a straight line going along the edge of the base platform. This straight line is usually parallel to a horizontal longitudinal axis of the moving base platform and parallel to velocity vector.

It is known, that the moment of force equals the product of multiplying the force by a shoulder. Hence, the module of the overturning moment equals

$F_{u H} \cdot H=m \cdot \frac{v^{2}}{R} \cdot H$

The moment which, on the contrary, tends to keep a moving exosystemin a 'normal' position thus trying to press the exosystemto a horizontal base platform is the moment of force of the whole system against a straight line going along the edge of the base platform.

Taking up the width of the base platform equal $\mathrm{L}$, we get shoulder $\mathrm{L} / 2$. Therefore the module of the stabilizing moment equals.

$P \cdot \frac{L}{2}=m g \cdot \frac{L}{2}$

The exosystem moving on a horizontal surface will not fall sideways if the overturning moment does not overcome the stabilizing moment, i.e. if the condition of lateral stability is met.

$m \cdot \frac{v^{2}}{R} \cdot H \leq P \cdot \frac{L}{2}$

Hence, the velocity should not exceed the maximum allowable one.

$v \leq v_{\max }=\sqrt{\frac{g L R}{2 H}}$

E.g., condition (3) will be metunder the increase in the maximum allowable velocity by $10 \%$ and simultaneous increase in the width of base platform $L$ by $21 \%$.

It is necessary to notice, that under sharp turns (sharp change of a direction of movement), i.e. under small radii of the trajectory curvature, the condition of lateral stability will be violated and the exosystemoverturning sideways will take place.

As minimally allowable radius of trajectory curvature is 
proportional to squaredvelocity, a minimally allowable radius of turn increases 9 times given 3 times increase in the velocity, e.g., from $2 \mathrm{~km} / \mathrm{h}$ up to $6 \mathrm{~km} / \mathrm{h}$. Hence, it is necessary to cautiously reducecontrol actionsto operate the exosystemevasion from an obstacle.

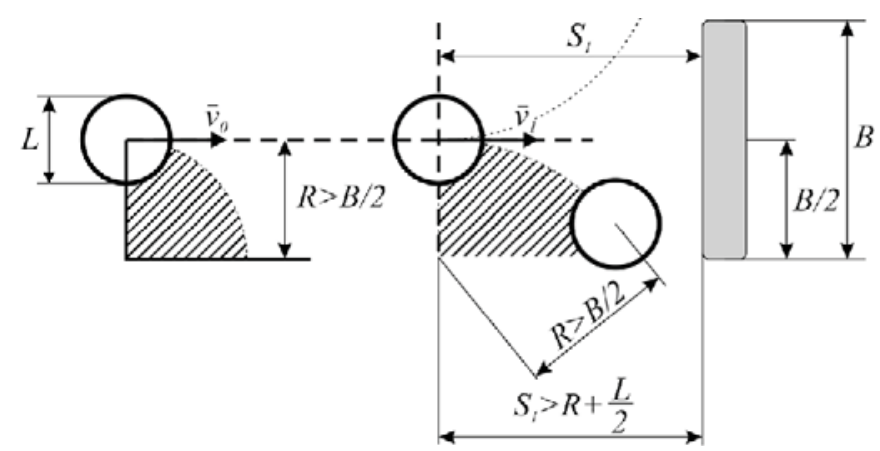

Figure 5: Lateral instability zone

Fig. 5 shows the case when the turning radius exceeds half of the obstacle width $R>B / 2$.

Thus, the first dangerous zone is found out,which is the zone of lateral instability. Entering it should be avoided, i.e.the exosystem cannot turn sharply, as well as create too small radius of trajectory curvature, as the condition of lateral stability will be violated and the exosystemwill fall sideways (Fig. 5).

If we assume, that during the movement of a base platform along a straight line it can come across an obstacle with $B$ width, it is not always possible for the platform to avoid collision. Let us define the dimensions of the first dangerous zone.

One way to avoid collision is to continue the movement, evading from an obstacle, but not turning sharply (with a small radius of trajectory curvature), i.e. not entering a zone of lateral instability. For this purpose, on the one hand, it is necessary 'to have time to evade', i.e. during the evasion the radius of trajectory curvature should be more than a half of the obstacle width increased for a half width of the base platform (in order not to touch an obstacle by the body side), that is

$R>\frac{B}{2}+\frac{L}{2}$

At the same time it is necessary that the exosystem did not fall sideways in going round the obstacle. To meet the condition of lateral stability the radius of trajectory curvature should be more than a minimally allowable one

$R>R_{\min }=\frac{2 \cdot H \cdot v^{2}}{g \cdot L}$

Hence, it is still time to fulfil the 'maneuver of evasion' when distance $\mathrm{S} 1$ to an obstacle is not short and conditions (5) and (6) are met simultaneously. An attempt to come to an obstacle nearer than the limiting distances in conditions (5) and (6) with a subsequent attempt to evade from an obstacle will lead to serious undesirable consequences: either to collision with an obstacle, or to exosystemoverturning sideways. In an 'extreme case', when the radius of trajectory curvature appears equal half of the obstacle width and the distance to obstacle S2 equals minimally allowable radius of trajectory curvature increased for a half width of the base platform(Fig. 6), the maneuver of evasion helps to avoid collision, however the final direction of movement becomes perpendicular to direction of movement at the beginning of the maneuver.

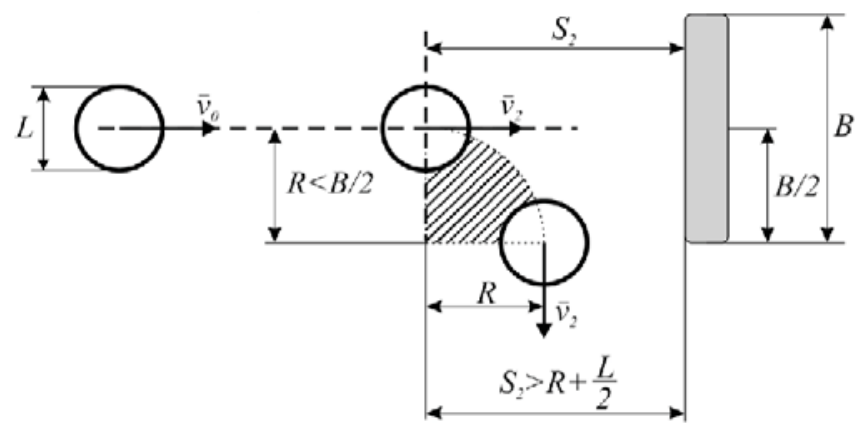

Figure 6: Lateral instability zone

Thus, a moving person in the exoskeleton must avoid entering the first dangerous zone where the condition of lateral stability is violatedleading to lateral overturning. Another way to avoid collision is to continue movingalong straight line to an obstacle, braking and stopping in front of an obstacle. For this purpose the velocity should not be too high, and distance (S1orS2) to an obstacle should be no less than braking path ST. Let us find the size of the braking path. Let the exosystemmodelled by a material point goes rectilinearly with initial velocity $\mathrm{v} 3$ along the deceleration segment, reduces the velocity under friction force FTp and stops (Fig. 7). Let us place the beginning of coordinates into point $O$ where deceleration begins. Then the original coordinate equals zero, and original velocity equals v3.

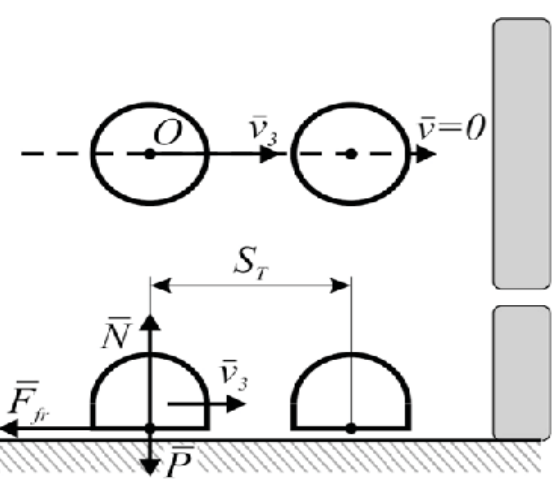

Figure 7: Definingstopping length and linear dimension of the second dangerous zone (top view and side elevation) 
Directing coordinate axis in the direction of rectilinear movement, we use Newton's second law and find projections of forces to the axis, where $\mathrm{f}$ is friction force, $\mathrm{N}$ is normal response equal in modulus to gravity force $\mathrm{mg}$.

$$
\begin{aligned}
& m \bar{a}=\bar{F}_{T P}+\bar{N}+\bar{P}, \\
& m a=-F_{T P}=-f \cdot N=-f \cdot m g
\end{aligned}
$$

Taking into account the fact, that acceleration equals the first derivative from velocity against timet, it is possible to obtaina differential equation with separable variables, integrate it and find a constant of integration, using the initial condition for velocity (given $t=0 \mathrm{v}=\mathrm{v} 3$ ). This will result in the law of velocity change

$$
v=v_{3}-f \cdot g t
$$

using which it is possible to calculate the time of movement up to stopping

$t^{*}=\frac{v_{3}}{f \cdot g}$

Taking into account the fact, that velocity equals the first derivative from coordinate against time, it is again possible to obtain a differential equation, integrate it and calculate a constant of integration, using the initial condition for coordinate (givent $=0 x=0$ ). Thiswill result in the law of motion

$x=-f \cdot g \cdot \frac{t^{2}}{2}+v_{3} \cdot t$

Substituting the time of movement up to a stop into it, we shall obtain a braking path

$S_{T}=\frac{v^{2}}{2 f g}$

In case of small factors of friction the braking path becomes long. If the velocity increases twice, the braking path increases four times.

Thus, we obtain the second dangerous zone where, for the sake ofmovement safety, the linear sectionintercepted between an obstacle and an exosystem moving to it(opposite coordinate axis $\mathrm{Ox}$ ) should be sufficient for both maneuvers (the first maneuver is evasion from an obstacle along the radius of curvature no less than minimally allowable againstthe condition of lateral stability, and the second one is braking and stoppingin front of an obstacle).

\section{CONCLUSIONS}

1. Under sharp turns (sharp change of movement direction), i.e.under small radii of trajectory curvature, the condition of lateral stability will be violated and exosystem sideways overturning will take place.

2. To increase the maximum allowable velocity by 10 $\%$, which excludesexosystem sideways overturning (the height of center of gravityHof the whole system and radiusRof trajectory curvature being unchanged), it is necessary to increase widthLof a base platform by $21 \%$.

3. The velocity of the exosystemshould not exceed the maximum allowable velocitydetermined against a condition of lateral stability.

4. In case of movement of the person with an exoskeletonit is necessary to avoid entering the first dangerous zone where the condition of lateral stability is violated and there is danger of lateral overturning.

5. When coming across an obstacle, the person with an exoskeleton should avoid entering the second dangerous zone where there is no opportunity to stop not having collidedwith an obstacle, or to evade from an obstacle not having overturned sideways.

6. Avoiding entering both the first and the second dangerous zones is a necessary condition for implementingthe 'stationary property of a system', i.e. the property of an exosystemsafe movement, i.e.the property of safe movementof the person ina medical exoskeleton.

\section{ACKNOWLEDGEMENT}

The given work is executed within the framework of implementation of the complex project on creation of hitech manufacture on Creation of hi-tech manufacture of multipurpose robotized medical exoskeleton (REM), code 2017-218-09-1807, authorized by the decision of the Government of the Russian Federation No. 218 of 9 April 2010.

\section{REFERENCES}

1. Ivanov D. et.al., (2017). Robotized exoskeleton must be!.The Engineer (VSUT), 1927(4), 12.

2. LoskutovYu.V. et. al., (2017). Computer simulation of regular walking based on the kinematic analysis of movement and the synthesis of exoskeleton control algorithms of regular walking on the basis of kinematic analysis of movements and synthesis of algorithms of exoskeleton control. Vestnik of the Volga State University of Technology. Ser.: Radio engineering and infocommunication systems, 3, 47-60. doi:10.15350/2306-2819.2017.3.47

3. AndrianovD.Yu., ,\&Fishchenko P.A., (2017). Dangerous zones form in case of a pilotless transport platform movement. Proceedings of the Volga State 
University of Technology. Ser.: Technological, 5, 209-306.

4. AndrianovD.Yu., ,\&Fishchenko P.A., (2016). Conditions of lateral stability of a pilotless transport platform moving on a horizontal surface. U: II All-Russia student conference Engineering stuff as a future of innovative economy of Russia, Yoshkar-Ola. Volga State University of Technology.8-13.

5. IFToMMdictonaries online, fromhttp://www.iftomm-terminology.antonkb.nl, accessed on 201806-02.

6. Raytheon XOS 2 Exoskeleton, Second-Generation Robotics Suit, United States of America, fromhttp://www.army-technology.com/projects/raytheon-xos-2-exoskeleton-us/, accessed on 2018-06-04.

7. NASA's Ironman-Like Exoskeleton Could Give Astronauts, Paraplegics Improved Mobility and Strength, fromhttp://www.nasa.gov/offices/oct/home/feature exoskeleton.html, accessed on 2018-06-04.

8. Eksobionics, fromhttp://eksobionics.com, accessed on 2018-07-04.

9. Introducing the: ReWalk Personal 6.0 System: The sixth generation ReWalk Personal Exoskeleton, fromhttp://rewalk.com, accessed on 2018-06-04.

10. Walking Assist, fromhttp://world.honda.com/Walking-Assist/introduction/index.html, accessed on 2018-06-04.

11. Panasonic Power Loader Light exoskeleton takes a load off your back, fromhttp://newatlas.com/ panasonic-power-loader-light-exoskeleton/25682/, accessed on 2018-06-04.
12. Cyberdyne, fromhttp://www.cyberdyne.jp/english/index.html, accessed on 2018-06-04.

13. France's slender Hercule exoskeleton is no lightweight, fromhttp://www.army-technology.com/features/featurefrench-hercule-robotic-exoskeleton, accessed on 2018-06-04.

14. Exoathlet, fromhttps://www.exoatlet.com/ru, accessed on 2018-06-04.

15. BorisovA.V., Konchina L.V., \&AbrosovYa.A., (2016). Control algorithm for maintenance of static vertical stability of an exoskeleton and a person in it under fluctuations of the person's position. Problems of the Russian society safety: Russian University of transport (MIIT), 2, 184-201.

16. VorochayevaL.Yu., YatsunA.S., \&Yatsun S.F., (2017). Control over exoskeleton quasistatic walking on the basis of expert system. SPIIRAS Proceedings, 3(52), 70-94.

17. Yatsun S.F., Savin S.I., YatsunA.S.,\&Mal'chikovA.V. (2016) Studying of an exoskeleton controlled movement on a frontal plane in the mode of balance restoration.Extreme robotics, 1, 236-245.

18. Dobrynin D.A. (2017) Principles of construction of a trained control system for an exoskeleton managementtask. Extreme robotics, 1, 297-301 